\title{
Monitorización de nanopartículas de oro mediante espectroscopia de absorción atómica de fuente continua de alta resolución con atomización electrotérmica
}

\author{
Raúl Garde, Martín Resano, Esperanza García \\ Grupo de Métodos Analíticos Rápidos mediante Técnicas Espectroscópicas (MARTE) \\ Instituto de Investigación en Ingeniería de Aragón (I3A) \\ Departamento de Química Analítica, Universidad de Zaragoza, Pedro Cerbuna 12, 50009, Zaragoza, Spain. \\ Tel. 843510-, e-mail: 612737@unizar.es
}

\begin{abstract}
Mediante la aplicación de una rampa de atomización lenta $\left(150^{\circ} \mathrm{C} \mathrm{s}^{-1}\right)$, ha sido posible la caracterización del tamaño medio de nanopartículas de oro (AuNPs) mediante espectroscopia de absorción atómica de fuente contínua de alta resolución con atomización electrotérmica (HR CS GFAAS) sin utilización de modificantes de matriz o etapas previas. El parámetro ultilizado para este fin es el tiempo del máximo de aparición de la señal, también llamado atomization delay, el cual es posible relacionarlo mediante calibración lineal con el tamaño de partícula. Esta metodología ha permitido la identificación de enlace entre nanoclusters de oro (AuNCs) y colina oxidasa (ChOx), de interés en sensores y la proporción óptima entre estos dos.
\end{abstract}

\section{Introducción}

La nanotecnología es uno de los campos de mayor desarrollo en los últimos años para diversas aplicaciones como sensores, medicina o ciencia de materiales por su relación superficie-volumen y sus propiedades derivadas de esta característica. Es por ello que el desarrollo de metodologías que aúnen la posibilidad de cuantificar y caracterizar la muestra al mismo tiempo es de gran interés.

La técnica utulizada en este trabajo es la espectroscopia de absorción atómica de fuente continua de alta resolución con atomización electrotérmica (HR CS GFAAS), previamente a este estudio se demostró la aplicación de esta técnica para la diferenciación entre iones plata y nanopartículas de este elemento [1] [2], debido a la diferente temperatura de atomización entre estas dos especies.

El objetivo del estudio es evaluar las capacidades de la técnica para diferenciar muestras con iones de oro y AuNPs, diferenciar entre tamaños de AuNPs y establecer la formación de enlace covalente entre colina oxidasa (ChOx) y AuNCs, utilizado en sensores.

\section{Experimental y Resultados}

Todos los experimentos se llevaron a cabo en un espectrómetro de absorción atómica de fuente continua (contrAA 700, Analytik Jena, Jena, Alemania), equipado con un atomizador de grafito de calentamiento transversal. Las muestras de nanopartículas de oro en suspensión de tamaños 5, 20, 50, 80 y $100 \mathrm{~nm}$ fueron adquiridas de Nanocomposix (Praga, República Checa). $\mathrm{HCl}$ y agua purificada obtenida de un sistema Mili-Q se utilizaron para diluir las muestras a la concentración deseada para su posterior análisis previa sonicación de las suspensiones de nanopartículas.

En los estudios previos sobre plata, queda patente la diferente temperatura de atomización dependiendo del tamaño de partícula de la muestra, necesitando mayores temperaturas para atomizar partículas de mayor tamaño. Mediante la optimización de la rampa de atomización y la temperatura de atomización, fue posible obtener una separación entre las señales de oro iónico y AuNP de $50 \mathrm{~nm}$ con una separación de más de 1 segundo. En la tabla 1 se indica el programa optimizado aplicado a lo largo de todos los experimentos.

El análisis de las diferentes nanopartículas mostró como cada una de ellas atomizaba más tarde conforme aumentaba el tamaño de partícula. En la figura 2 se representa el tiempo de aparición del máximo de señal frente al tamaño medio medido mediante TEM proporcionado por Nanocomposix. Se puede observar que al representar los diferentes tamaños aparecen dos regiones lineales; una primera de mayor sensibilidad desde los iones de oro hasta las nanopartículas de 20 nanómetros y la segunda de 
menor sensibilidad desde $20 \mathrm{~nm}$ hasta la muestra de $100 \mathrm{~nm}$. [3]

Mediante el uso de este programa se analizaron unas muestras preparadas de nanoclusters unidos covalentemente a colina oxidasa para aplicación en sensores. El análisis comparativo de las señales de las muestras de NCs, NCs en presencia de ChOx y NCs-ChOx tras hacerlos reaccionar (figura 2). Se puede observar tres regiones de aparición de picos asociados a diferentes interacciones NC-proteína; una primera región en torno a los $9 \mathrm{~s}$ asociada a efectos de matriz inherente a la presencia de la proteína en la muestra (aparece en todas las muestras con proteína), una segunda región sobre 10 segundos en las que sólo aparece pico en las muestras donde se ha llevado a cabo reacción, y una tercera en torno a los $12 \mathrm{~s}$ asociada a NCs de oro libres. El análisis de diversas proporciones NCChOx (2-1, 3-1, 6-1) mostró la aparición de pico en esta tercera región a partir de la proporción 3-1, lo que indica la presencia de NCs libres.

\begin{tabular}{ccccc}
\hline $\begin{array}{c}\text { Programa de } \\
\text { Temperatura }\end{array}$ & $\begin{array}{c}\text { Temperatura } \\
/{ }^{\circ} \mathrm{C} \mathrm{s}^{-1}\end{array}$ & $\begin{array}{c}\text { Rampa } \\
/{ }^{\circ} \mathrm{C} \mathrm{s}^{-1}\end{array}$ & $\begin{array}{c}\text { Tiempo de } \\
\text { espera / }\end{array}$ & $\begin{array}{c}\text { Flujo de gas } \\
\text { Ar/ L min }\end{array}$ \\
\hline Secado & 90 & 3 & 20 & 2 \\
Secado & 110 & 5 & 10 & 2 \\
Pirólisis & 300 & 50 & 30 & 2 \\
Pirólisis & 300 & 0 & 5 & 0 \\
Atomización & 2200 & 150 & 10 & 0 \\
Limpieza & 2450 & 500 & 4 & 2 \\
\hline
\end{tabular}

Tabla 1. Programa optimizado utilizado.

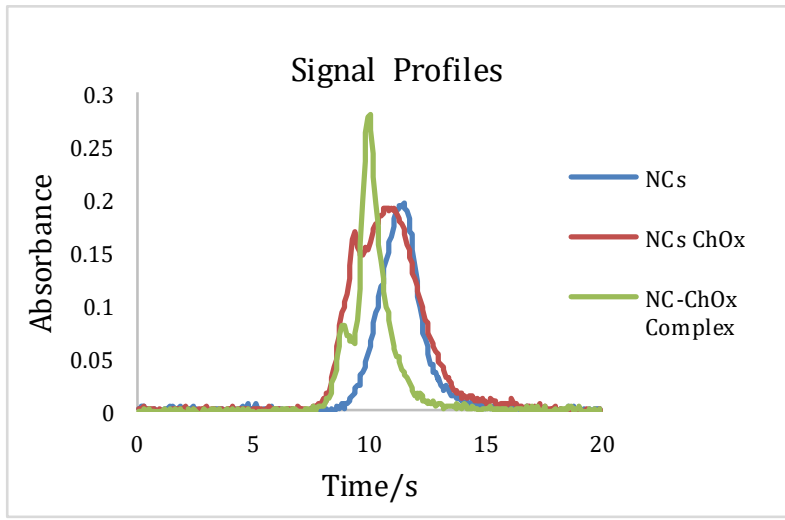

Fig. 2. Señales correspondientes a las muestras de nanoclusters de oro (azul), nanoclusters de oro en presencia de colina oxidasa (rojo) y nanoclusters unidos covalentemente a colina oxidasa (verde).

\section{Conclusiones}

La aplicación de rampas de atomización lentas permite la caracterización de tamaño de nanopartículas mediante calibración lineal, presentando dos rangos lineales; hasta $20 \mathrm{~nm}$ y de ahí hasta $100 \mathrm{~nm}$. Este método ha permitido a su vez caracterizar la unión covalente y la proporción entre nanoclusters de oro y colina oxidasa para su aplicación en biosensores.

\section{REFERENCIAS}

[1]. F. Gagné, P. Turcotte and C. Gagnon, Anal. Bioanal. Chem., 2012, 404, 2067-2072.

[2]. N. S. Feichtmeier and K. Leopold, Anal. Bioanal. Chem., 2014, 406, 3887-3894.

[3]. M. Resano, E. Garcia-Ruiz and R. Garde, $J$. Anal. At. Spectrom., 2016, 31, 2233-2241.

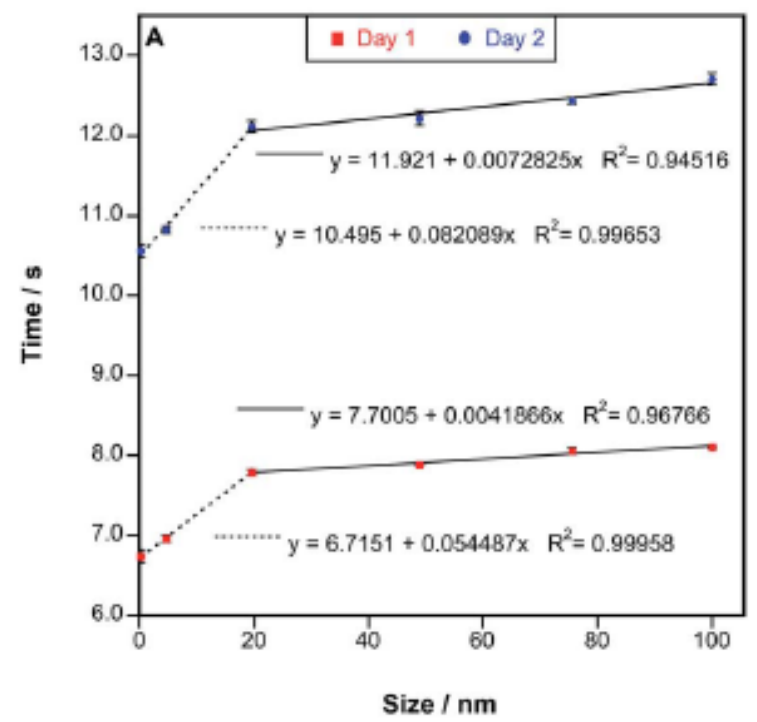

Fig. 1. Atomization delay frente al tamaño de partícula. 